\title{
Cluster Randomised Trials - Ivermectin Repurposing for Covid-19 Treatment of Outpatients with Mild Disease in Primary Health Care Centers
}

\section{Rossana Elena Chahla}

Ministry of Health

Luis Medina Ruiz

Ministry of Health, SI.PRO.SA

\section{Teresa Mena}

Ministry of Health, SI.PRO.SA

Yolanda Brepe

Ministry of Health, SI.PRO.SA

Paola Terranova

Ministry of Health, SI.PRO.SA

\section{Eugenia Silvana Ortega}

Ministry of Health, SI.PRO.SA

Guillermo Gabriel Barrenechea

Ministry of Health, SI.PRO.SA

Daniel Gustavo Goroso

Mogi das Cruzes University

María de los Ángeles Peral de Bruno ( $\square$ mperal@fm.unt.edu.ar)

Ministry of Health, SI.PRO.SA

\section{Research Article}

Keywords: COVID-19, Ivermectin, Outpatient care, Mild-stage, Health Primary Care.

Posted Date: March 2nd, 2022

DOl: https://doi.org/10.21203/rs.3.rs-495945/v2

License: (1) (i) This work is licensed under a Creative Commons Attribution 4.0 International License.

Read Full License 


\section{Abstract}

Background: The emergence of COVID-19 requires alternative treatments. This study aims to evaluate the therapeutic intervention with ivermectin in outpatients with COVID-19 mild disease, to increase discharge and prevent the progression to moderate or severe disease.

Methods: Randomized Trial in an overall ratio 1:1, $\mathrm{n}=254$. The subjects were divided into experimental $(E G: n=110)$ and control groups placebo (CG: $n=144)$. The $E G$ received ivermectin orally $0.6 \mathrm{mg} / \mathrm{kg}$ weight in two doses. All participants were by physical examination COVID-19 diagnosed with negative RTPCR at the beginning and the end of protocol. Differences between the variables were determined using the Chi-square test $(p<0.05)$. The contagion risk (Odds Ratio) was calculated using software STATA.

Results: Both groups were similar in age, sex, and comorbidities. A significant reduction in the percentage of participants with symptoms (PPS) was observed in the EG and CG when the clinical evaluation of symptoms was performed from $5^{\text {th }}$ to $9^{\text {th }}(p=0.0005)$. When the clinical evaluation was performed from $10^{\text {th }}$ to $14^{\text {th }}$ day there was no significant difference. A higher proportion of outpatient discharge was observed in EG (98.2\%) vs. CG (86.1\%) ( $p$-Value $=0.0007)$. EG showed 8 times more chance of receiving discharge than CG (OR 8.71, 95\% Cl: $1.99-38 \cdot 12, p=0 \cdot 004)$. The treatment effect with ivermectin to obtain discharge from outpatient care was analyzed by the logistic regression. Then, the chance to obtain outpatient discharge was independent of variables sex, age, and comorbidities.

Conclusion: Treatment with ivermectin in outpatients care with mild disease of COVID-19 managed to slightly reduce PPS. The treatment with ivermectin could significantly prevent the evolution to serious stages since the EG did not present any patient with referral to critical hospitalization.

Retrospective Clinical Registration: ClinicalTrial.gov Identifier NCT04784481; Trial registration date: 03-05-2021. Last updated: 04-26-2021.

\section{Introduction}

Since the beginning of the pandemic, despite drastic containment measures, the spread of COVID-19 has threatened to collapse health systems around the world, and also had devastating socioeconomic consequences worldwide [1]. The impact of the coronavirus pandemic has disproportionately affected developing countries whose economies are less able to cope with the new challenges imposed [2].

International health authorities have focused on the rapid diagnosis and isolation of patients, as well as the search for therapies capable of counteracting the most serious effects of the disease, which constitute approximately $15 \%$ of the cases according to WHO $[3,4]$. Due to the number of infections continues to increase exponentially, the development of vaccines and new antiviral therapies are crucial. While vaccines have been approved for their use, difficulties in responding to high demand and logistical complexities that accentuate potential inequality must be considered. Hence, repurposing 
pharmaceuticals remains an important strategy to tackle COVID-19. In this context, the repositioning of drugs currently available on the market with established safety profiles that are implemented on other therapeutic indications, based on solid preclinical studies, is imperative. This is a pragmatic strategy, a faster and cheaper option compared to the new drug development that has proven successful for many medications and can be a key tool in emergencies such as the current one that requires a quick action [58]. This strategy becomes more relevant in those economies that do not have the necessary resources for the development of new therapies, as in the case of Latin-American countries. In addition, the COVID-19 anti-vaccine movements that have recently become known in these countries.

Ivermectin is a broad-spectrum antiparasitic agent that has been shown to have antiviral activity against a wide range of viruses. Caly et al. (2020) suggested that ivermectin's nuclear transport inhibitory activity may be effective against SARS-CoV-2(9). In line with this study, numerous clinical trials, especially from developing countries, are evaluating the potential of ivermectin against COVID-19 with results that are not conclusive yet regarding its efficacy and safety [10-14].

In Argentina's health system, the emergency of COVID-19 requires the urgent development of strategies to avoid the impact of the disease on our population, prevent the saturation of the health system and allow us to carry out adequate treatments to reduce the mortality of the disease. In this context, our health system considered the study of the repositioning of ivermectin as a strategy to prevent the progression to moderate or severe stages of COVID 19 disease since it is a safe drug, which is available in our environment and with antecedents in other health systems in the world of its use both in treatment and prevention.

This study aims to evaluate the therapeutic intervention with ivermectin in outpatients with COVID-19 mild disease, to increase discharge and prevent the progression to moderate or severe disease.

Primary Outcome. Increase discharge from outpatient care with COVID-19 mild disease.

Secondary Outcomes. Decrease the PPS from outpatient's care.

\section{Material And Methods}

\subsection{Sample Size}

Sample size was determined by the test comparing two proportions [15]. It considered the following parameters to the bilateral test: $n=30,000$ Total operating area population for services network to Primary Care; $95 \%$ confidence level, $3 \%$ precision, $5 \%$ proportion, $n=201$ sample size without loss, $20 \%$ expected proportion of losses. The sample size calculated was $n$

$=252$ participants.

\subsection{Participants}


The total group $n=254$ enrolled outpatients care from the urban area-department heads and peri-urban area of the City San Miguel de Tucumán. The study was conducted between September 2020 to January 2021. The health coverage service was administered by the Health System of the State of Tucumán (SI.PRO.SA, Tucumán, Argentina). The people who agreed to participate in the study gave their informed consent before starting the study (Independent Ethical Committee / Health Research Directorate, Ministry of Health, Tucumán Government, Argentina, IRB: 054/2020 in accordance with the ReNIS, National Register in Health Research, Argentina /0032). The clinical trials registry number is NCT04784481. This study conforms to all CONSORT guidelines and reports the required information accordingly (see Supplementary Checklist).

\subsection{Inclusion criteria}

- Over 18 years of age of any sex.

- Outpatients infected by SARS-CoV-2 confirmed by positive RT-PCR test.

- Women of childbearing age with a negative pregnancy test.

- Mild disease-patients with two or more of the following symptoms: fever less than $38.5^{\circ} \mathrm{C}$ and higher than $37.5^{\circ} \mathrm{C}$ according to Ministry of Health, Argentina [16], isolated diarrheal episodes, hyposmia or hypogeusia, mild desaturation (between 96 and 93\%), dyspnea, polyarthralgia, persistent headache, abdominal pain, erythema of the kidney, nonspecific rash.

\subsection{Exclusion Criteria}

- Hypersensitivity or allergy to ivermectin.

- Pregnant or lactating.

- Children or adolescents under 18 years of age.

- Patients with neurological pathology, renal insufficiency, hepatic insufficiency.

- Weight less than $40 \mathrm{~kg}$.

- Patients with concomitant use of drugs that act on GABA, barbiturate and benzodiazepine receptors.

- Patients who have not completed / signed the informed consent.

\subsection{Randomization and masking}

Cluster-randomized trials. Participant centers depend on the Public Health System of Tucumán, Argentina. Each of the health centers (cluster of health centers) was randomly assigned to receive or not the intervention under study. The conglomerate of outpatients randomly assigned to the Experimental Group (EG) and Control Group (CG) belongs to the urban and peri-urban areas from Tucumán, and the selection of the centers was carried out considering a homogeneous socioeconomic profile in relation to the operating areas in which it is organized the Public Health Systems. Participants were recruited prior to cluster randomization. Staff of each assistance center knew what intervention was being implemented as 
well as patients. Data processing group was blind to analyze the database. The difference between the number of patients shown in the CG (110) and GA (144) is explained by the fact that the of health centers present some differences in the areas of population coverage, which cannot be ignored since the clusters were randomized after recruitment of patients.

Figure 1 shows the CONSORT flow diagram. Note that 331 patients were initially assessed for eligibility, $\mathrm{n}$ $=280$ recruited, and allocated to this study $n=254$ distributed in EG $(n=110)$, and CG $(n=144)$. The clinical evaluation of symptoms considered for a 1 st time frame was carried out from 5 th to 9 th day, this is since the effects of the treatment can manifest around 7 days $( \pm 2)$ and whereas it is the period during which the disease may progress to more serious stages, being evaluated in this instance in EG $(n=98)$ and CG $(n=51)[17,18]$. Patients were reviewed too in a $2 n d$ time frame: from 9th to 14 th day EG $(n=12)$, and $C G(n=79)$.

\subsection{Intervention Protocol}

The EG received protocol ivermectin orally 4 tablets of $6 \mathrm{mg}=24 \mathrm{mg}$ every 7 days for 4 weeks plus symptomatic treatment (500mg paracetamol every 6 or $8 \mathrm{~h}$, no more than 4 tablets daily; $100 \mathrm{mg}$ aspirin, 1 tablet per day with breakfast; $150 \mathrm{mg}$ Ranitidine, 1 tablet in the morning, and 1 tablet at night). The CG received only symptomatic treatment. Patients with comorbidities continued with the basic medication for the underlying pathology (see description in results section). All participants were evaluated by physical examination and COVID-19 infection was diagnosed with positive RT-PCR. Clinical evaluation of symptoms was carried throughout the study period. Enrolled subjects completed symptom questionnaires (including reporting of any adverse effects of treatment), physical examinations and remote clinical telemedicine follow-up and received outpatient discharge 4 weeks after the start of the intervention.

The 10-category ordinal scale recommended by the WHO was used to classify patients according to clinical patient state: ambulatory mild disease, moderate disease, severe disease [19].

\section{Security definitions}

An Adverse Event (AE) was defined as any medical event, sign, symptom, or disease temporarily associated with the use of the medication, which could occur in the subjects enrolled in the study [20].

\section{Adherence to treatment}

WHO defines adherence to treatment as compliance with it; that is, taking the medication according to the dosage of the prescribed schedule; and persistently taking the medication over time [21]. We quantify adherence to treatment through weekly controls that include drug administration and a clinical questioning which includes the report of adverse events. Adhesion tests like Hermes, Morisky and Green have not been used, since they have been designed for treatment of chronic diseases with daily drug intake [22]. Coordinators in charge of each health care center were responsible for the accompaniment during the trial. 
Categorical variables were summaries with frequencies and percentages, and continuous variables with median and interquartiles. Differences between the categorical variables were estimated using the Chisquare test. The proportion test was applied to compare the proportion of participants with symptoms. Logistic regression was used to model the odds of medical release by sex, comorbidities and age (Odd Ratio: OR). The level of statistical significance was reached when $p<0 \cdot 05$. Calculations were performed using STATA $11 \cdot 2$.

\section{Results}

\subsection{Demographic profile}

In total, $\mathrm{n}=254$ were enrolled by cluster-randomized for this study. The subjects were divided into experimental ( $E G: n=110$; median $=40 \cdot 0$ years old, $\min =18 \cdot 0, \max =75 \cdot 0,56$ female) and control groups (CG: $n=144 ;$ median $=36$ years old, $\min =18 \cdot 0, \max =71 \cdot 0,77$ female), $p$-Value $>0 \cdot 05$. Table 1 shows the demographic profile and descriptions of comorbidity for the experimental and control group. 
Table 1

Demographic Profile

\begin{tabular}{|c|c|c|}
\hline Variables & $\begin{array}{l}\text { Experimental Group } \\
(n=110)\end{array}$ & $\begin{array}{l}\text { Control Group } \\
(n=144)\end{array}$ \\
\hline \multicolumn{3}{|l|}{ Demographic profile } \\
\hline Median Age (in years) & 40 & 36 \\
\hline Interquartile Range (IQR) & {$\left[\mathrm{IQR}_{25}: 19 ; \mathrm{IQR}_{75}: 53\right]$} & {$\left[\mathrm{IQR}_{25}: 29 ; \mathrm{IQR}_{75}: 48\right]$} \\
\hline \multicolumn{3}{|l|}{ Gender $-n^{\circ}$. (\%) } \\
\hline Female & $56(50.91 \%)$ & $77(53.47 \%)$ \\
\hline Male & $54(49.09 \%)$ & $67(46.53 \%)$ \\
\hline \multicolumn{3}{|l|}{ Co-morbidities - $\mathrm{n}^{\circ}$. (\%) } \\
\hline HTA & $14(12.73 \%)$ & $18(12.50 \%)$ \\
\hline DBT & $9(8.18 \%)$ & $7(4.86 \%)$ \\
\hline Obesity & $8(7.27 \%)$ & $4(2.78 \%)$ \\
\hline$>60$ years & $12(10.91 \%)$ & $7(4.86 \%)$ \\
\hline Asthma & $1(0.91 \%)$ & $2(1.39 \%)$ \\
\hline
\end{tabular}

There were no significant differences in baseline characteristics between the experimental and control group. It was observed the following frequency of HTA, DBT, and obesity in the EG relationship to CG; $14: 18,9: 7$, and 8:4, respectively, however, this difference was not significant. Of the GC patients, $20 \%$ (29/144) had some comorbidity, of which 65.5\% (19/29) were under medical treatment. 31\% (9/29) reported taking ENALAPRIL, 20.7\% (6/29) METFORMIN and 13.8\% (4/29) LOSARTAN. Of the EG patients, $30.9 \%(34 / 110)$ had some comorbidity of those referred to at work, of these patients, $38.2 \%(13 / 34)$ reported being under medical treatment. 14.7\% (5/34) took ENALAPRIL, 8.8\% (3/34) METFORMIN, 5.9\% $(2 / 34)$ LOSARTAN, and $8.8 \%(3 / 34)$ other medicines.

\subsection{Decreased number of patients with symptoms at 5th - 9th day, and posterior}

Figure 2 demonstrates a greatest decrease of PPS from the 5th to 9th day, considering the relation between the number of patients with symptoms and the number of patients clinically evaluated: in EG $48 / 98$ and in CG 40/51. Proportion test presented significant differences inter-group $p=0 \cdot 0005$. Intragroup the difference was significant same in both group $(p=0.0001)$. Additionally, the difference is higher 
in EG $(51 \%)$ vs. CG $(21 \cdot 6 \%)$. After the date reported, from 10th to 14 th day the medical examination did not show a significant difference between groups. In the 2 nd time frame was observed significative different only intra-group EG $(p=0 \cdot 0187)$.

The association test between the sex variable in both groups showed that there are no significant differences between 1 st time frame and 2 nd time frame. The same happened when the age variable was included.

Table 2 shows the clinical profile of COVID-19 symptoms in the 1 st time frame. The symptoms were divided in two categories: I) systemic symptoms and, II) upper airways symptoms.

Table 2

Percentage of number symptoms description 1st time frame.

\begin{tabular}{|lccllll|}
\hline \multicolumn{2}{c}{ Control Group } & \multicolumn{5}{c|}{ Experimental Group } \\
\hline Symptoms & Enrollment & $\begin{array}{c}\text { 1st Time } \\
\text { Frame }\end{array}$ & $p$ & Enrollment & $\begin{array}{c}\text { 1st Time } \\
\text { Frame }\end{array}$ & $p$ \\
\hline I. Percentage Systemic Symptoms in COVID-19 & & & & \\
\hline Fever & 38.2 & 7.5 & 0.0000 & 55.5 & 8.3 & 0.0000 \\
\hline Diarrhea & 25.0 & 5.0 & 0.0000 & 21.8 & 20.8 & 0.0145 \\
\hline Polymyoarthralgia & 13.9 & 2.5 & 0.0000 & 40.9 & 16.7 & 0.0000 \\
\hline Headache & 46.5 & 22.5 & 0.0000 & 43.6 & 16.7 & 0.0000 \\
\hline Body pain & 34.7 & 2.0 & 0.0000 & 17.3 & 6.3 & 0.0006 \\
\hline Abdominal pain & 9.7 & 2.5 & 0.0007 & 4.5 & 10.4 & 0.98 \\
\hline Dyspnea & 6.9 & 2.5 & 0.0062 & 6.4 & 8.3 & 0.353 \\
\hline Tiredness & 16.7 & 5.0 & 0.0000 & 8.2 & 6.3 & 0.079 \\
\hline II. Percentage Upper airway symptoms in COVID-19 & & & & \\
\hline $\begin{array}{l}\text { Taste and/or smell } \\
\text { disturbance }\end{array}$ & 41.0 & 55.0 & 0.0000 & 34.5 & 20.8 & 0.0000 \\
\hline Odynophagia & 16.0 & 2.5 & 0.0000 & 15.5 & 0 & $*$ \\
\hline Cough & 17.4 & 27.5 & 0.0175 & 19.1 & 20.8 & 0.0438 \\
\hline
\end{tabular}

There was a decrease in the percentage of systemic and upper airways symptoms reported in the medical examination in both groups (Table 2). It was observed in the systemic symptoms that in both groups there is a favorable response to conventional treatment and to ivermectin treatment. However, ivermectin 
treatment was found to be more effective in alleviating upper airway symptoms, with a significant drop in cough ( $p$-Value $=0.05)$. The proportion test showed that the difference in symptom reduction was in favor of EG with regard to taste and/or smell disorder $(p$-Value $=0.00001)$

\subsection{Discharge of clinic at 28 days after enrollment}

The second finding of this study was the number of patients who received medical release at 28 days after enrollment (Fig. 3)

Because of the treatment, the EG had a higher medical discharge than the CG. Proportion was EG: $\mathrm{n}=$ 108/110; CG: $n=124 / 144, p$-Value $=0.0007$. It was observed that there are no differences in achieving medical discharge when adjusting for the age of the patient, both for the median age and for the distribution of interquartile. Similar results were observed for sex distribution. It should be noted that no deaths were reported and no patient left the intervention. Moreover, most of the participants were discharged from EG $(n=108)$, and for CG $(n=8)$, the rest continued under treatment and observation.

\subsection{Bivariate analysis and Logistic regression}

Bivariate analysis showed 8 times more chance of receiving medical release in EG than CG (OR

$=7.99,95 \%, 1.64-38.97, p$-Value $=0.003)$. When adjusting for age, sex and comorbidities the odds of medical release was maintained in EG $(\mathrm{OR}=8 \cdot 71, \mathrm{Cl}=[1 \cdot 99,38 \cdot 12] ; p=0 \cdot 004)$ (Table 3).

Table 3

Logistic regression

\begin{tabular}{|lllll|}
\hline Variables & Odds Ratio & \multicolumn{2}{l}{ Cl (95\%) } & $\boldsymbol{p}$ \\
\hline Ivermectin & 8.71 & 1.99 & 38.12 & 0.004 \\
\hline Comorbidities & 0.80 & 0.45 & 1.43 & 0.455 \\
\hline Sex & 2.07 & 0.81 & 5.26 & 0.127 \\
\hline Age & 1.02 & 0.98 & 1.06 & 0.237 \\
\hline
\end{tabular}

\section{Discussion}

This study was designed to evaluate the potential of ivermectin as a repositionable drug, for the treatment of mild cases of COVID-19. Ivermectin has shown an effect on clinical manifestations of COVID-19, including a decrease in the percentage of symptoms reported in the medical examination and an increase in the chance for medical release in EG.

Currently, many studies about ivermectin and its potential against SARS-CoV-2 are complete or in development. A pilot study that evaluates the effect of early treatment with ivermectin for COVID-19, 
sheds some light on the potential of ivermectin in a tendency to lower viral loads and lower IgG titers [23]. Other work, either from Argentina shows a faster viral clearance in treated participants [13]. Chaccour et al. reported that patients treated with ivermectin experienced a significant diminution of $50 \%$ anosmia/ hyposmia than those in the placebo group (76 vs. 158 patient-days of anosmia/hyposmia) [23]. The ivermectin group also reported $30 \%$ fewer coughs (68 vs. 97 patient-days of cough). However, in this study, there were no major differences between ivermectin and placebo in the reported patient days of fever, general malaise, headache, or nasal congestion [23].

In line with these results, in our study, outpatients report a significant drop in the percentage of upper airway symptoms in COVID-19 (taste and/or smell disturbance, odynophagia, cough), see Table 2. Concomitantly, the results reported here show that the use of ivermectin produces a decrease in the number of symptoms reported by patients, such as fever and diarrhea, but above all, a significant decrease in taste and smell loss, which is related to the effects of viral load on upper air vials in patients with mild COVID-19. No patient from either group progressed to severe disease.

Recently, Lopez Medina et al. published a study of ivermectin concerning the time resolution of symptoms in mild patients [24]. Although they do not recommend the use of ivermectin as a treatment for COVID-19, there are some similarities (and differences) with the results of the present study. We agree that there are no significant differences between both groups at 14 days (see Fig. 2), but our main finding shows that the effect of treatment is observed between 5 and 8 days after the patient starts treatment. This difference may be due to the administration of the dose, which in our case is weekly and not daily. The clinical follow-up of the patients was carried out in person in the Primary Health Centers, which is more accurate concerning symptom recording.

There are results that provide evidence of the potential benefit of early intervention with ivermectin for the treatment of patients diagnosed with mild stages of COVID-19, as Elgazzar et al. trial [14]. Many studies present the potential of ivermectin for a viral load reduction, as has been suggested by Caly et al. in vitro. This could have the potential effect on disease progression and spread. [13, 14, 23, 25]

A single-center prospective clinical trial performed in 167 patients with mild to severe COVID- 19 from Argentina, found that none of the mild or moderate cases of COVID-19 who received the experimental treatment with ivermectin were hospitalized, and only one patient died (0.59\%) [26]. In México a comparative effectiveness study was performed among patients with laboratory-confirmed SARS-CoV-2 infection. The experimental group received a TNR4. TNR4 consists of four drugs administered orally to COVID-19 cases with mild or moderate symptoms:

(1) ivermectin, 12 MG single dose; (2) azithromycin $500 \mathrm{mg}$ for 4 days; (3) montelukast, $60 \mathrm{mg}$ on the first day and then $10 \mathrm{mg}$ between days 2 to 21 ; and (4) acetylsalicylic acid, $100 \mathrm{mg}$ for 30 days. This study indicated that the TNR4 significantly increases the likelihood of full recovery within 14 days after the onset of symptoms, and decreases the risk of hospitalization or death among ambulatory cases of COVID-19. [27] 
The addition of ivermectin to standard care can be effective for the treatment of COVID-19 patients with significant reductions in mortality and duration hospital stay compared to Hydroxychloroquine plus standard treatment [14]. Early use of ivermectin is very useful for controlling COVID-19 infections, improving cytokines storm and prophylaxis of frontline health care as well as household contacts [14].

Our study has sizable limitations. The absence of a placebo group is due to the lack of funding from a sponsor and the need to guarantee treatment to the entire population. The sample size, although representative, is too small to obtain conclusive results. It's a descriptive study of clinical follow-up at 28 days without report of adverse events which it would be beneficial to evaluate in future works. Or study demonstrates similar benefits with other studies, and taken together, these results are encouraging for further study about repurposing ivermectin for the treatment of COVID-19, considering that it is an inexpensive drug and is accessible in the local pharmaceutical industry (Argentina). We suggest new clinical intervention studies in our region and other countries that may show the effect of the IVER compound in mild-stage outpatients.

\section{Conclusion}

Treatment with ivermectin in a population of outpatients with COVID-19 mild disease managed to significantly reduce the number of symptoms when clinical evaluation was performed from 5 th to 9th. Subsequent clinical evaluation from day 10 th to 14 th did not show significant differences.

Participants received ivermectin had a greater chance of medical release vs. CG ( $p=0.0007)$. This proposed treatment brings additional benefits in relation to the improvement in the patient's clinical condition, without this having an impact on adherence.

\section{Declarations}

\section{Ethics approval and consent to participate}

The people who agreed to participate in the study gave their informed consent before starting the study. The protocol was approved by Independent Ethical Committee / Health Research Directorate, Ministry of Health, Tucumán Government, Argentina, IRB: 054/2020 in accordance with the ReNIS, National Register in Health Research, Argentina /0032.

\section{Consent for publication}

"Not applicable"

\section{Data availability}

The datasets generated during and/or analyzed during the current study are available from the corresponding author on reasonable request. The database collected for the study will be made available 
on request for scientific interests. Information available includes: investigation protocol, informed consent and database of trial with ID numbers to protect patient's identity.

\section{Conflict of Interests}

The authors did not receive any monetary compensation for this work. They declare that they have no known competing financial interests or personal relationships that could have appeared to influence the work reported in this paper.

\section{Funding}

The Ministry of Public Health. Tucumán, Argentina participated in the design of the study and the collection, analysis and interpretation of the data, as well as in the drafting of the manuscript.

\section{Authors' contributions}

ESO supervised the database. GGB, ESO and DGG contributed with the data processing and contributed to the statistical analysis. ESO, DGG and MPB were responsible for writing the manuscript. TM, YB and PT contributed to data collection. REC and LMR were the institutional managers to carry out the work. MPB supervised the project.

\section{Acknowledgements}

All the authors are grateful for the collaboration of the health and administrative personnel of the Primary Cares Centers: Adolfo de la Vega, San Martín, 25 de Mayo, Alberdi, Alderete, Nuestro Sr. del Milagro, Vial III, Delia Fernandez Palma, Esquina, Fernando P. Riera, Bella Vista Hospital, Los Ralos Hospital, Lomas de Tafí, Los Pocitos, Villalonga Policlinic, Santa Ana, and Active Search Center for Febrile and Symptomatic (in Spanish language: "Centro de Búsqueda Activa de Febríles y Asintomáticos"). The authors thank Ms. Emma Lis Garat (Dirección Investigación en Salud, SI.PRO.SA, Tucumán, ARG) and Dr. Augusto Bellomio (INSIBIO UNT, Tucumán, ARG) for critical reading of the manuscript. We thank the Ministry of Public Health of Tucuman in whose centers the study was carried out.

\section{References}

1. Carvallo H, Hirsch R, Alkis P, Contreras V. Study of the Efficacy and Safety of Topical Ivermectin + lota-Carrageenan in the Prophylaxis against COVID-19 in Health Journal of Biomedical Research and Clinical Investigation. 2020; 2(1).

2. Sataloff RT, Johns MM, Kost KM. The unequal impact of the coronavirus pandemic: evidence of seventeen development countries [Internet]. 2020. Available from: 
https://publications.iadb.org/publications/english/document/The-Unequal-Impact-of-theCoronavirus-Pandemic-Evidence-from-Seventeen-Developing-Countries.pdf

3. Wu Z, McGoogan JM. Characteristics of and Important Lessons from the Coronavirus Disease 2019 (COVID-19) Outbreak in China: Summary of a Report of 72314 Cases from the Chinese Center for Disease Control and Prevention. JAMA - Journal American Medical Association. 2020; 323(13):1239-42.

4. What we know about long-term effects of COVID-19. The latest on the COVID- 19 global situation and long-term sequelae. 2020;(September). Available from: https://www.who.int/docs/defaultsource/coronaviruse/risk-comms-updates/update-36- long-term-symptoms.pdf?sfvrsn=5d3789a6_2

5. Chong CR, Sullivan, New uses for old drugs. Nature [Internet]. 2007; 448(7154):645-

6. Available from: https://doi.org/10.1038/448645a

7. Ashburn TT, Thor KB. Drug repositioning: identifying and developing new uses for existing drugs. Nature Reviews Drug Discovery [Internet]. 2004; 3(8):673-83. Available from: https://doi.org/10.1038/nrd1468

8. Liu Z, Fang H, Reagan K, et al. In silico drug repositioning-what we need to know. Drug Discovery Today. Elsevier Ltd. 2013; 18: 110-5.

9. Dyall J, Coleman CM, Hart BJ, et al. Repurposing of clinically developed drugs for treatment of Middle East respiratory syndrome coronavirus Antimicrobial Agents and Chemotherapy. 2014; 58(8):4885-93.

10. Caly L, Wagstaff KM, Jans DA. Nuclear trafficking of proteins from RNA viruses: potential target for antivirals? Antiviral Research. 2012; 95(3):202-6.

11. Chahla RE, Medina Ruiz L, Ortega ES., et al. Prophylaxis Covid-19 in Healthcare Agents by Intensive Treatment with Ivermectin and lota-carrageenan (Ivercar-Tuc) American Journal of Therapeutics [Internet]. 2021; 28 (5). Available from: https://clinicaltrials.gov/ct2/show/NCT04701710? term=ivermectin\&cond=Covid19\&cnt ry=AR\&city=Tucuman\&draw=2\&rank=1

12. Cassará Ivermectina asociada a iota-Carragenina aplicada localmente en la cavidad bucal, en la profilaxis de la enfermedad COVID-19 en el personal de salud. Estudio IVERCAR01. 2020; 1-23.

13. Ministerio de Salud Pública de la Provincia de Tucumán. Protocolo de Investigación I.D.D.E.A - TUC. 2020.

14. Krolewiecki A, Lifschitz A, Moragas M, et al. Antiviral Effect of High-Dose Ivermectin in Adults with COVID-19: A Pilot Randomised, Controlled, Open Label, Multicentre Trial. SSRN Electron J [Internet]. 2020; Available from: 2139/ssrn.3714649

15. Elgazzar A, Hany B, Youssef SA. Efficacy and Safety of Ivermectin for Treatment and prophylaxis of COVID-19 Pandemic. Research Square [Internet]. 2020; 1-9. Available from: https://doi.org/10.21203/rs.3.rs-100956/v1

16. Glueck DH. Sample Size Calculations in Clinical Research 2 nd edition by CHOW, S.- , SHAO, J., and WANG, H. Biometrics. 2008; 64(4):1307-8. 
17. Ministerio de Salud de la Nación Argentina. Criteria for defining COVID-19 cases [Internet]. 2020. Available from: https://argentina.gob.ar/salud/coronavirus- COVID-19/definicion-de-caso

18. Zhou F, Yu T, Du R, et al. Clinical course and risk factors for mortality of adult inpatients with COVID19 in Wuhan, China: a retrospective cohort Lancet [Internet]. 2020; 395(10229):1054-62. Available from: http://dx.doi.org/10.1016/S0140-6736(20)30566-3

19. Rubin JE, Crowe SE. COVID-19 Symptoms: Longitudinal Evolution and Persistence in Outpatient Settings. Annals of Internal 2020; 172(1): ITC1-14.

20. Marshall JC, Murthy S, Diaz J, et al. A minimal common outcome measure set for COVID-19 clinical research. Lancet Infectious Diseases. 2020; 20(8): e192-7.

21. WHO-adverse reaction terminology (WHO-ART). In: Dictionary of Pharmaceutical Vienna: Springer Vienna; 2009. p. 192-3.

22. Osterberg L, Blaschke T. Adherence to Medication. The New England Journal of Medicine. 2005; 353(5):487-97.

23. Rodríguez Chamorro MÁ, García-Jiménez E, Amariles P, Rodríguez Chamorro A, Faus MJ. Revisión de tests de medición del cumplimiento terapéutico utilizados en la práctica clínica. Atención Primaria. 2008; 40(8):413-7.

24. Chaccour C, Casellas A, Blanco-Di Matteo A, et al. The effect of early treatment with ivermectin on viral load, symptoms and humoral response in patients with non-severe COVID-19: A pilot, doubleblind, placebo-controlled, randomized clinical EClinicalMedicine. 2021; 32:100720.

25. López-Medina E, López P, Hurtado IC, et al. Effect of Ivermectin on Time to Resolution of Symptoms Among Adults with Mild COVID-19: A Randomized Clinical Trial. Jama [Internet]. 2021;1-10. Available from: http://www.ncbi.nlm.nih.gov/pubmed/33662102

26. Caly L, Druce JD, Catton MG, Jans DA, Wagsta KM. The FDA-approved drug ivermectin inhibits the replication of SARS-CoV-2 in vitro. Antiviral Research. 2020; 178:104787.

27. Carvallo H, Hirsch R, Farinella ME. Safety and efficacy of the combined use of ivermectin, dexamethasone, enoxaparin and aspirin against COVID medRxiv the preprint server for health sciences. 2020; https://doi.org/10.1101/2020.09.10.20191619

28. Lima-Morales R, Méndez-Hernández P, Flores YN, et al. Effectiveness of a multidrug therapy consisting of ivermectin, azithromycin, montelukast and acetylsalicylic acid to prevent hospitalization and death among ambulatory COVID-19 cases in Tlaxcala, Mexico. International Journal of Infectious Diseases. 2021; 105: 598-605.

\section{Figures}




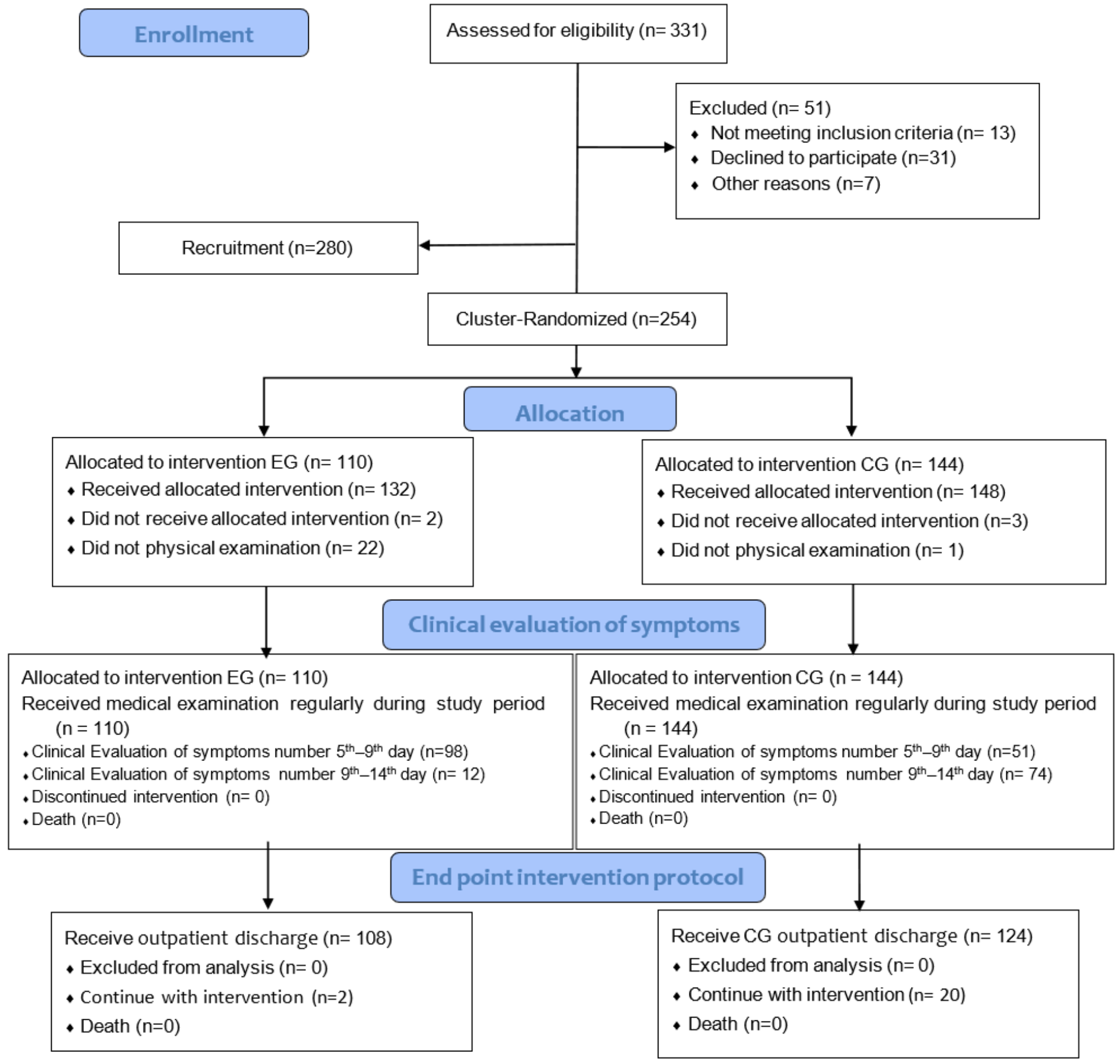

\section{Figure 1}

Consort flow diagram. 


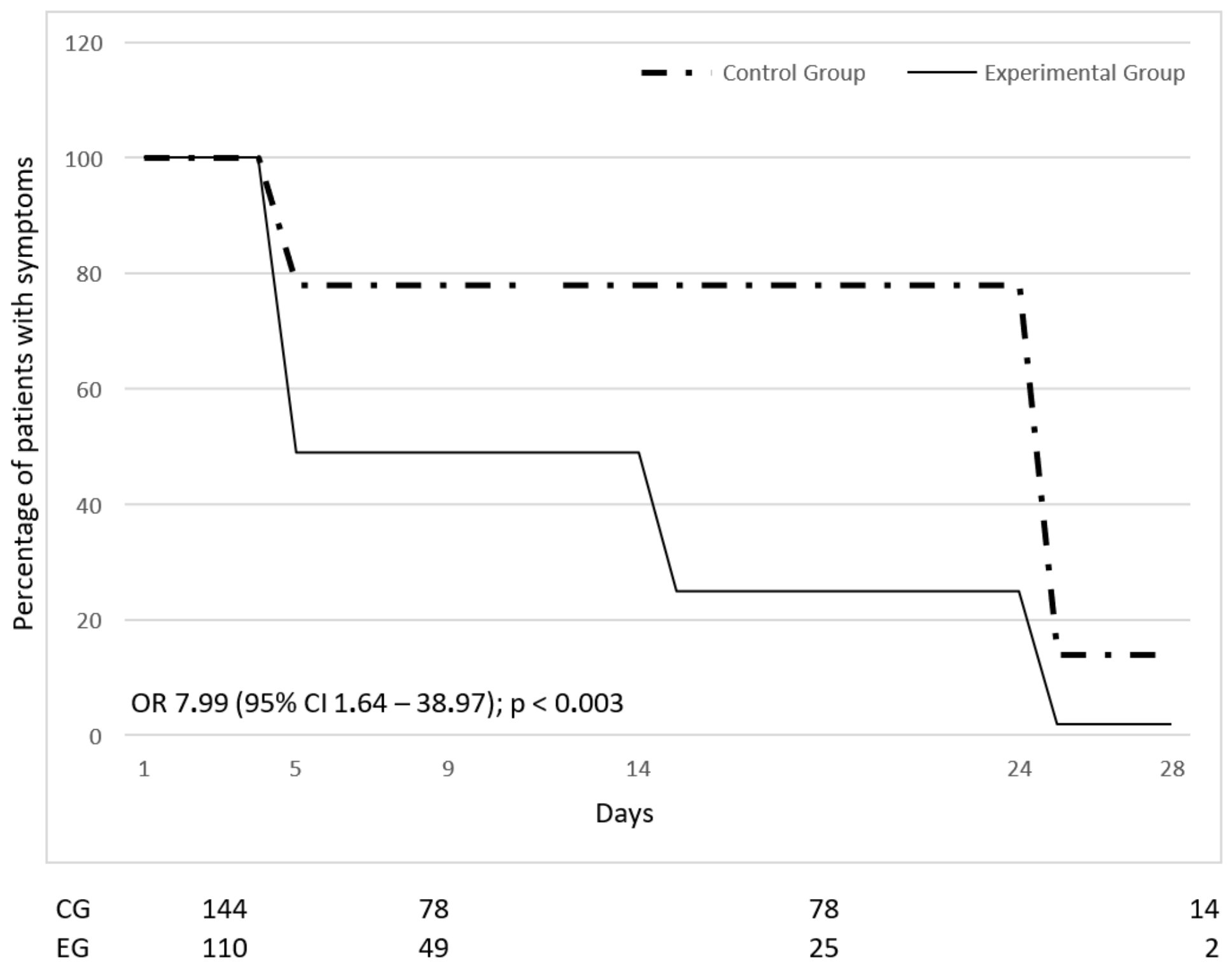

Figure 2

Kaplan-Meier plot. Percentage of patients with symptoms: i) Enrollment, ii) $1^{\text {st }}$ time frame from $5^{\text {th }}$ to $9^{\text {th }}$ day; iii) $2^{\text {nd }}$ time frame from $10^{\text {th }}$ to $14^{\text {th }}$ day 
100

98.2

98

96

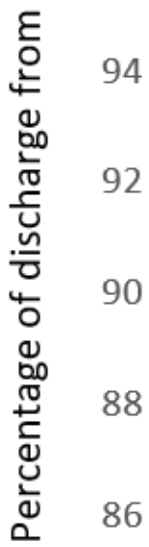

84

82

80

86.1

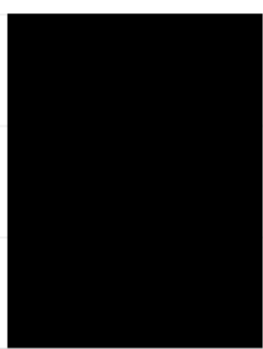

Control Group

Experimental Group

Figure 3

Outpatient discharge at 28 days after enrollment 